\title{
Attribute Metadata A Proposed Schema for Evaluating Federated Attributes
}

Paul A. Grassi

Naomi B. Lefkovitz

Ellen M. Nadeau

Ryan J. Galluzzo

Abhiraj T. Dinh

This publication is available free of charge from: https://doi.org/10.6028/NIST.IR.8112

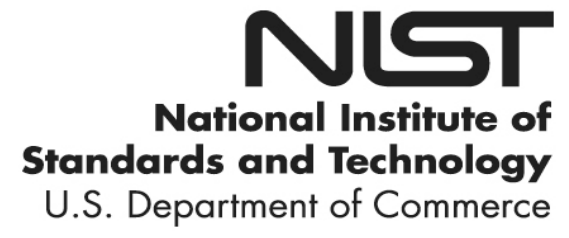




\title{
Attribute Metadata \\ A Proposed Schema for Evaluating Federated Attributes
}

\author{
Paul A. Grassi \\ Naomi B. Lefkovitz \\ Ellen M. Nadeau \\ Applied Cybersecurity Division \\ Information Technology Laboratory \\ Ryan J. Galluzzo \\ Abhiraj T. Dinh \\ Deloitte \& Touche LLP \\ Rosslyn, $V A$
}

This publication is available free of charge from:

https://doi.org/10.6028/NIST.IR.8112

January 2018

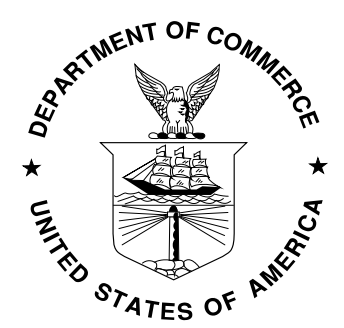

U.S. Department of Commerce Wilbur L. Ross, Jr., Secretary

National Institute of Standards and Technology Walter Copan, NIST Director and Under Secretary of Commerce for Standards and Technology 


\title{
National Institute of Standards and Technology Internal Report 8112 \\ 44 pages (January 2018)
}

This publication is available free of charge from:

https://doi.org/10.6028/NIST.IR.8112

Certain commercial entities, equipment, or materials may be identified in this document in order to describe an experimental procedure or concept adequately. Such identification is not intended to imply recommendation or endorsement by NIST, nor is it intended to imply that the entities, materials, or equipment are necessarily the best available for the purpose.

There may be references in this publication to other publications currently under development by NIST in accordance with its assigned statutory responsibilities. The information in this publication, including concepts and methodologies, may be used by federal agencies even before the completion of such companion publications. Thus, until each publication is completed, current requirements, guidelines, and procedures, where they exist, remain operative. For planning and transition purposes, federal agencies may wish to closely follow the development of these new publications by NIST.

Organizations are encouraged to review all draft publications during public comment periods and provide feedback to NIST. Many NIST cybersecurity publications, other than the ones noted above, are available at http://csrc.nist.gov/publications.

\section{Comments on this publication may be submitted to:}

\author{
National Institute of Standards and Technology \\ Attn: Applied Cybersecurity Division, Information Technology Laboratory \\ 100 Bureau Drive (Mail Stop 2000) Gaithersburg, MD 20899-2000 \\ Email: nsticworkshop@nist.gov
}

All comments are subject to release under the Freedom of Information Act (FOIA). 


\title{
Reports on Computer Systems Technology
}

The Information Technology Laboratory (ITL) at the National Institute of Standards and Technology (NIST) promotes the U.S. economy and public welfare by providing technical leadership for the Nation's measurement and standards infrastructure. ITL develops tests, test methods, reference data, proof of concept implementations, and technical analyses to advance the development and productive use of information technology. ITL's responsibilities include the development of management, administrative, technical, and physical standards and guidelines for the cost-effective security and privacy of other than national security-related information in federal information systems.

\begin{abstract}
This NIST Internal Report contains a metadata schema for attributes that may be asserted about an individual during an online transaction. The schema can be used by relying parties to enrich access control policies, as well as during run-time evaluation of an individual's ability to access protected resources. Attribute metadata could also create the possibility for data sharing permissions and limitations on individual data elements. There are other possible applications of attribute metadata, such as evaluation and execution of business logic in decision support systems or associated with devices or non-person entities; however, the metadata contained herein is focused on supporting an organization's risk-informed authorization policies and evaluation for individuals.
\end{abstract}

\section{Keywords}

access control; assertions; attributes; attribute metadata; attribute schema metadata; attribute values; attribute value metadata; authorization; federation; identity; identity federation; information security; metadata; privacy; risk; risk management; security; trust.

\section{Acknowledgments}

The authors would like to thank Josh Freedman for his significant contributions to this report, as well as Sean Brooks for his considerate inclusion of privacy related content. In addition, we would like to thank Anil John and the Federal Identity, Credential, and Access Management (FICAM) Attribute Tiger Team for their leadership in developing the initial set of attribute metadata necessary for federal systems. Finally, we express significant gratitude to Darran Rolls of SailPoint Technologies, Inc., as well as Gerry Gebel and David Brossard of Axiomatics, for their insightful review of this report. 
Executive Summary

This NIST Internal Report proposes attribute schema metadata and attribute value metadata as part of an overall schema intended to convey information about a subject's attribute(s) to allow for a relying party (RP) to:

- Obtain greater understanding of how the attribute and its value were obtained, determined, and vetted;

- Have greater confidence in applying appropriate authorization decisions to subjects external to the domain of a protected system or data;

- Develop more granular access control policies;

- Make more effective authorization decisions;

- Manage rules about the processing of data more effectively; and

- Promote federation of attributes.

This document defines a set of optional elements to support cross-organization confidence in attribute assertions as well as the semantics and syntax required to support interoperability. The schema contains two core components, attribute schema metadata and attribute value metadata which, along with their suggested elements, are described below:

- Attribute Schema Metadata (ASM) - Metadata for the attribute itself, not the specific attribute's value. For example, this metadata may describe the format in which the attribute will be transmitted, such as that height will always be sent in inches regardless of what the actual value may be (e.g., height= 72). This schema in Table 1 provides a set of attribute metadata from which to choose when constructing and executing an attribute sharing agreement (often called trust-time) for their inclusion.

Table 1 - Attribute Schema Metadata

\begin{tabular}{|l|l|l|}
\hline \multicolumn{2}{|c|}{ Metadata } & \multicolumn{1}{c|}{ Description } \\
\hline Description & $\begin{array}{l}\text { An informative description of } \\
\text { the attribute }\end{array}$ & Any \\
\hline Allowed Values & $\begin{array}{l}\text { A defined set of allowed } \\
\text { values for the attribute }\end{array}$ & Any \\
\hline Format & $\begin{array}{l}\text { A defined format in which the } \\
\text { attribute will be expressed }\end{array}$ & Any \\
\hline Verification Frequency & $\begin{array}{l}\text { The frequency at which the } \\
\text { Attribute Provider will re- } \\
\text { verify the attribute }\end{array}$ & Any \\
\hline
\end{tabular}




\section{Metadata}

Data Processing
Description

Describes the basis for processing attributes and attribute values

\section{Recommended Values}

Any

- Attribute Value Metadata (AVM) - These elements focus on the asserted value for the attribute. Following the same example as above, the attribute value would be the actual height. A possible AVM for the height could be the name of the originating organization that provisioned the height, for example the Department of Motor Vehicles (DMV) in the subject's home state. This schema in Table 2 provides a set of AVM and proposed values for those metadata fields.

Table 2 - Attribute Value Metadata

\begin{tabular}{|c|c|c|}
\hline $\begin{array}{l}\text { Metadata } \\
\text { Element }\end{array}$ & Description & Values \\
\hline Origin & $\begin{array}{l}\text { The name of the entity that issues or } \\
\text { creates the initial attribute value }\end{array}$ & $\begin{array}{l}-<\text { Origin’s Name }> \\
\text {-“None” }\end{array}$ \\
\hline Provider & $\begin{array}{l}\text { The name of the entity that is } \\
\text { providing the attribute }\end{array}$ & $\begin{array}{l}-<\text { Provider’s Name> } \\
\text {-“None” }\end{array}$ \\
\hline Pedigree & $\begin{array}{l}\text { Description of the attribute value's } \\
\text { relationship to the authoritative } \\
\text { source of the value }\end{array}$ & $\begin{array}{l}\text {-“Authoritative” } \\
\text {-“Sourced” } \\
\text {-“Self-Asserted” } \\
\text {-“Derived” }\end{array}$ \\
\hline Verifier & $\begin{array}{l}\text { The entity that verified the attribute's } \\
\text { value }\end{array}$ & $\begin{array}{l}\text {-“Origin" } \\
\text {-"Provider” } \\
\text {-"Not Verified" }\end{array}$ \\
\hline $\begin{array}{l}\text { Verification } \\
\text { Method }\end{array}$ & $\begin{array}{l}\text { The method by which the attribute } \\
\text { value was verified as true and } \\
\text { belonging to the specific individual }\end{array}$ & $\begin{array}{l}\text {-"Document Verification” } \\
\text {-"Record Verification” } \\
\text {-“Document Verification with } \\
\text { Record Verification” } \\
\text {-“Proof of Possession” } \\
\text {-“Probabilistic Verification” } \\
\text {-“Not Verified” }\end{array}$ \\
\hline
\end{tabular}




\begin{tabular}{|c|c|c|}
\hline $\begin{array}{l}\text { Metadata } \\
\text { Element }\end{array}$ & Description & Values \\
\hline $\begin{array}{l}\text { Last } \\
\text { Verification }\end{array}$ & $\begin{array}{l}\text { The date and time when the attribute } \\
\text { value was last verified as being true } \\
\text { and belonging to the specified } \\
\text { individual }\end{array}$ & No restrictions \\
\hline Last Refresh & $\begin{array}{l}\text { The date and time when the attribute } \\
\text { was last refreshed }\end{array}$ & No restrictions \\
\hline $\begin{array}{l}\text { Expiration } \\
\text { Date }\end{array}$ & $\begin{array}{l}\text { The date an attribute's value is no } \\
\text { longer valid }\end{array}$ & No restrictions \\
\hline $\begin{array}{l}\text { Date } \\
\text { Consented }\end{array}$ & $\begin{array}{l}\text { The date on which subject consent for } \\
\text { release of the attribute value was } \\
\text { acquired }\end{array}$ & No restrictions \\
\hline $\begin{array}{l}\text { Consent } \\
\text { Type }\end{array}$ & Indicates the type of consent & No restrictions \\
\hline $\begin{array}{l}\text { Acceptable } \\
\text { Uses }\end{array}$ & $\begin{array}{l}\text { Allowed use conditions for entities } \\
\text { that receive attributes }\end{array}$ & No restrictions \\
\hline $\begin{array}{l}\text { Cache Time } \\
\text { To Live }\end{array}$ & $\begin{array}{l}\text { The length of time for which an } \\
\text { attribute value may be cached }\end{array}$ & No restrictions \\
\hline $\begin{array}{l}\text { Data } \\
\text { Deletion } \\
\text { Date }\end{array}$ & $\begin{array}{l}\text { Indicates the date the attribute is to be } \\
\text { deleted from records }\end{array}$ & No restrictions \\
\hline Classification & $\begin{array}{l}\text { The security classification level of } \\
\text { the attribute }\end{array}$ & $\begin{array}{l}\text {-“Unclassified” } \\
\text {-“Controlled Unclassified” } \\
\text {-“Confidential” } \\
\text {-“Secret” } \\
\text {-“Top Secret” } \\
\text {-“Company Confidential” }\end{array}$ \\
\hline
\end{tabular}




\begin{tabular}{|c|c|c|}
\hline $\begin{array}{l}\text { Metadata } \\
\text { Element }\end{array}$ & Description & Values \\
\hline Releasability & $\begin{array}{l}\text { The restrictions regarding to whom } \\
\text { an attribute value may be released }\end{array}$ & $\begin{array}{l}\text {-“NATO” } \\
\text {-“NOFORN” } \\
\text {-“FVEY” } \\
\text {-“Public Release” } \\
\text {-“Externally Releasable for Business } \\
\text { Purposes” } \\
\text {-“Do Not Release” } \\
\text {-“None” }\end{array}$ \\
\hline
\end{tabular}

The schema in this document is intended to demonstrate the value of attribute schema and attribute value metadata in supporting U.S. federal government use cases. NIST envisions that the core set of metadata proposed here can serve as a library or menu from which both commercial and federal implementers can draw common semantics, syntaxes, and values to support their specific needs. This will serve as a starting point for the development of a metadata standard that can enable greater federation across markets and sectors.

Though this is a finalized document, this schema will be developed further in future revisions, based upon implementation feedback received by the community. 


\section{Table of Contents}

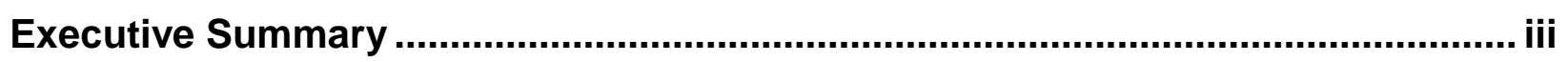

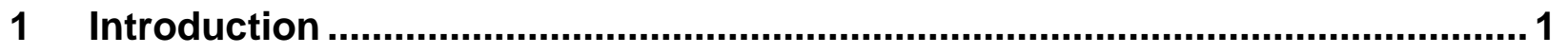

1.1 Purpose

1.2 Scope

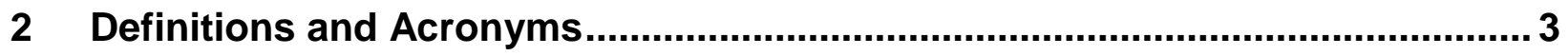

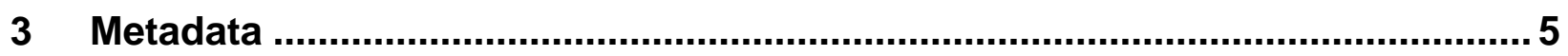

3.1 Attribute Schema Metadata …............................................................ 6

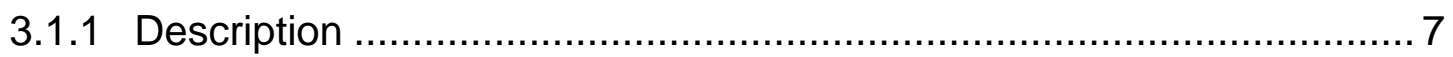

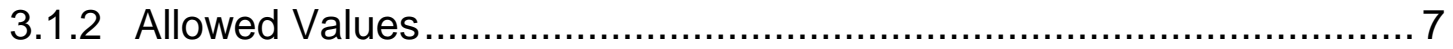

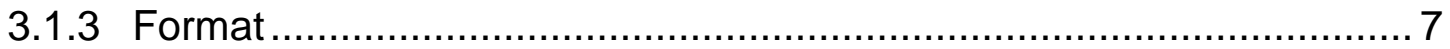

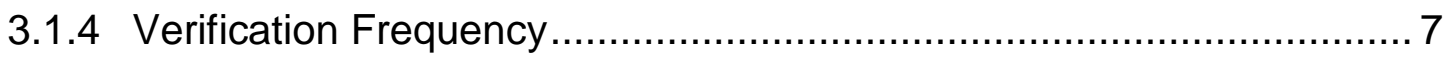

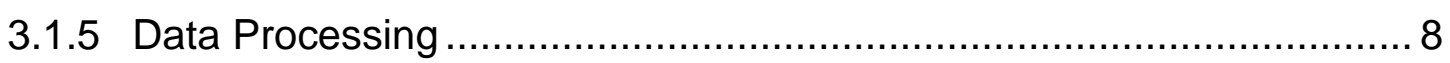

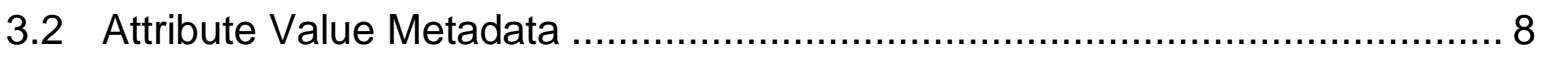

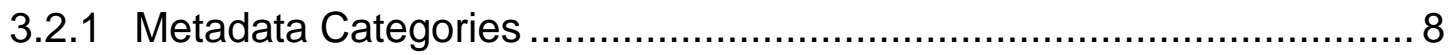

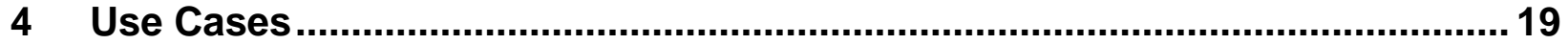

4.1 Federated Access to Classified Document in an Information Sharing

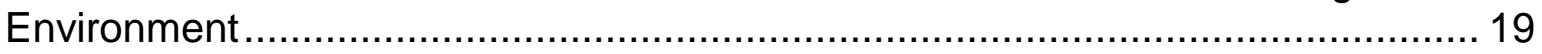

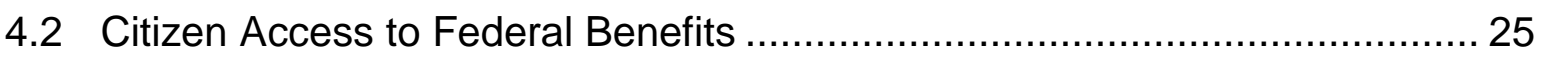

4.3 Law Enforcement Access to a Government Database............................... 28

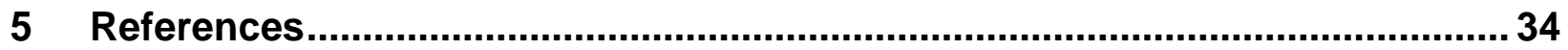

\section{List of Figures}

Figure 1 Trusted Access Using Attribute Metadata Assertion .................................... 5

\section{List of Tables}

Table 1 - Attribute Schema Metadata ...................................................................

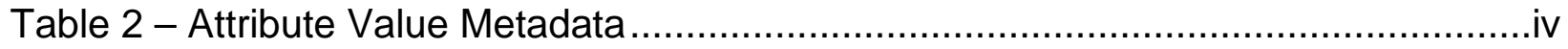

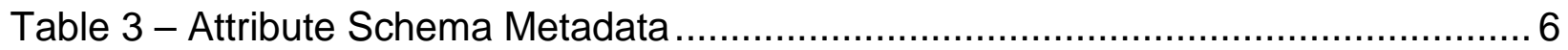

Table 4 - Categories of Attribute Value Metadata.................................................... 8

Table 5 - Distribution of Attribute Value Metadata Elements ........................................ 9 


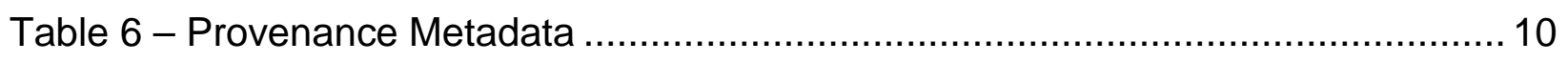

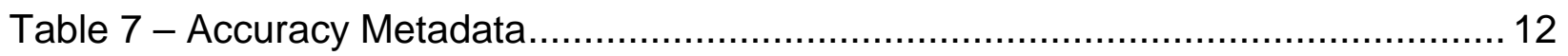

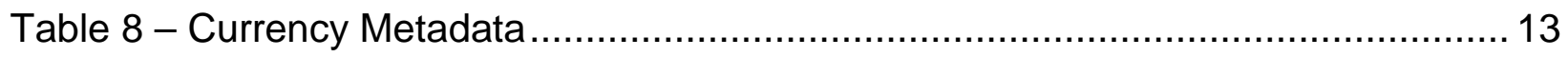

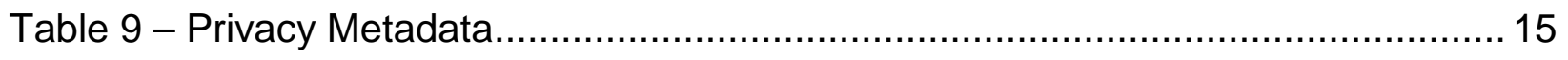

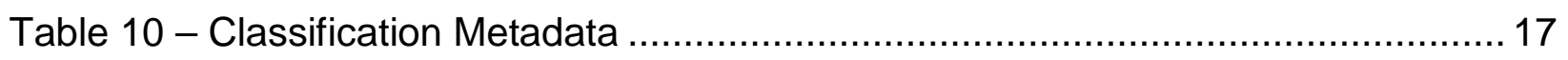

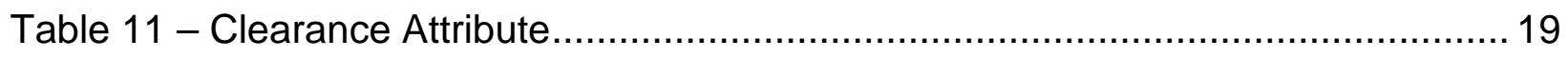

Table 12 - AVM to Support Access Control Decisions............................................... 20

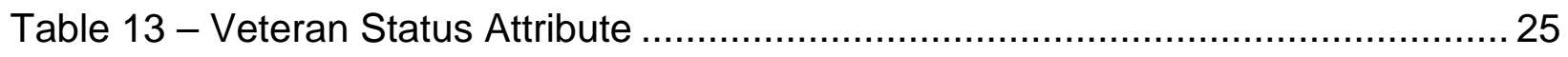

Table 14 - AVM to Support Enrollment Decisions …............................................... 26

Table 15 - Law Enforcement Officer Attributes ...................................................... 28

Table 16 - AVM to Support Federated Access Decisions.............................................29 


\section{Introduction}

Access control policy increasingly depends on evaluating the attributes of the individual or subject that is attempting to access a protected resource [Special Publication (SP) 800-162]. As enterprise domains continue to expand, architectures become further distributed, business relationships become more complex, and organizations increasingly depend on federated identities, methods are needed for evaluating externally asserted attributes to make the best and most appropriate authorization decision possible. Such mechanisms will increase the ability of organizations to consume attributes as well as enrich and enforce critical access control policies. At the "Advanced Identity Workshop: Applying Measurement Science in the Identity Ecosystem” (hereafter “workshop”) held at NIST in Gaithersburg on January 12 and 13, 2016, NIST proposed an initial set of attribute metadata as a step towards enabling greater federation and trust of identity attributes among identity ecosystem participants. This NIST Internal Report (NISTIR) represents a refined list of optional metadata that may be adopted when participating in a federated environment.

\subsection{Purpose}

This NISTIR proposes attribute schema metadata and attribute value metadata to convey information about a subject's attribute(s) to allow for a relying party (RP) to:

- Achieve greater understanding of how the attribute and its value were obtained, determined, and vetted;

- $\quad$ Promote greater confidence in applying appropriate authorization decisions to subjects external to the domain of a protected system or data (i.e., external users);

- Enable more effective authorization decisions; and

- Promote federation of attributes.

The model proposed in this document allows RPs to determine the most appropriate attribute metadata elements for a given transaction. In the future, it could serve as a foundation for an attribute confidence scoring structure to simplify further the process of aligning attribute based authorization decisions with the risk environment.

In addition, as a NISTIR, this document is intended to be treated as an "implementers' draft" so that developers and business owners can determine the efficacy and required adjustments of the attribute metadata elements. By issuing this as an implementers' draft, NIST seeks to obtain feedback on agencies' and industries' experiences with this approach in order to identify next steps, such as potentially transitioning this document to a NIST SP or a contribution to a private sector standards developer.

\subsection{Scope}

This NISTIR defines a set of optional elements of an attribute metadata schema to support crossorganization decision making, such as two executive branch agencies, in attribute assertions. It also provides the semantics and syntax required to support interoperability. NIST does not intend to make any of this schema required in federal systems and attribute-based information sharing. Rather, this schema represents a compendium of possible metadata elements to assist in risk- 
based decision making by an RP. This schema is focused on subjects (individual users); objects and data tagging, while related, are out of scope.

Specifically, this document addresses the following:

- Attribute Schema Metadata (ASM) - Metadata for the attribute itself, not the specific attribute's value. For example, this metadata may describe the format in which the attribute will be transmitted, such as that a subject's height will always be sent in inches regardless of what the actual value may be (e.g., height= '72'). This schema provides a set of attribute metadata from which to choose when establishing and executing an attribute sharing agreement (i.e., trust-time) and the rationale for their inclusion.

- Attribute Value Metadata (AVM) - These elements focus on the asserted value for the attribute. Following the same example as above, the attribute value would be the actual height (72). A possible AVM for the height could be the name of the originating organization that provisioned the height, for example the DMV in the subject's home state. This schema provides a set of AVM, proposed values for those metadata fields, and rationale for their inclusion.

- Use Cases - To demonstrate the applicability of the proposed metadata schema, this document also provides example use cases in which the application of the proposed schema would be used to support authorization decision making, thus allowing for greater confidence in federated identities and attributes.

- Example Assertions - Finally, this report includes example assertions illustrating what a technical implementation of the schema would look like leveraging standards such as Extensible Access Control Markup Language (XACML).

While the schema in this document is intended to demonstrate the value of attribute metadata in supporting U.S. federal government use cases, the ideal metadata schema could be used in both commercial and public sector implementations, thus serving as a foundation to enable greater federation across markets and sectors. Furthermore, NIST intends for the schema to be protocol and technology agnostic, thus capable of being supported across the spectrum of modern runtime access control architectures. 


\section{Definitions and Acronyms}

\section{Assertion}

A statement from an attribute provider to a relying party that contains identity attributes about a subject. Assertions may also contain authentication or other identity information about the subject.

\section{Attribute}

A reference of a named quality or characteristic inherent in or ascribed to someone or something.

\section{Attribute Based Access Control (ABAC)}

Access control based on attributes associated with subjects, objects, targets, initiators, resources, or the environment. An access control rule set defines the combination of attributes under which access may take place.

\section{Attribute Reference (or “Reference”)}

A statement asserting a property of a subject without necessarily containing authentication or other identity information, independent of format. For example, for the attribute 'birthday', a reference could be 'older than 18' or 'born in December'.

\section{Attribute Provider (AP)}

Manages and provides assertions of identity attributes to other relying and federated parties.

\section{Attribute Provider Statement (APS)}

A document that captures the security, privacy, data protection, and attribute management practices of a given attribute provider or party acting as an attribute provider for a given set of transactions.

\section{Attribute Schema Metadata (ASM)}

Data providing information about the context and structure of an attribute. See metadata.

\section{Attribute Value Metadata (AVM)}

Data describing an asserted value for an associated attribute.

\section{Authorization}

The decision to permit or deny a subject access to resources (e.g., network, data, application, services) based on the evaluation of access control policies. 


\section{Credential Service Provider (CSP)}

An entity that issues digital credentials to subjects and issues or registers authenticators for subjects' use. A CSP may be an independent third party, or may issue credentials for its own use. A CSP may provide and verify attributes or may assert attributes provided and verified by other entities.

\section{Federation}

A process that allows for the conveyance of identity attributes and authentication information across a set of networked systems.

\section{Identity Provider (IDP)}

A CSP in a federation that manages the subject's primary authentication credentials and issues assertions derived from those credentials.

\section{Metadata}

Structured information that describes, explains, locates, or otherwise makes it easier to retrieve, use, or manage an information resource. Metadata is often called data about information or information about information.

\section{Personally Identifiable Information (PII)}

Information that can be used to distinguish or trace an individual's identity, either alone or when combined with other information that is linked or linkable to a specific individual.

\section{Relying Party (RP)}

An entity that relies upon a subject's authenticator(s) and credentials or an IDP's assertion of a subject's identity, typically to process a transaction or to grant access to information or a system.

\section{Trust-time}

Refers to the process of establishing agreements between framework participants in order to develop metadata schema requirements consistent with community needs. 


\section{$3 \quad$ Metadata}

The term attribute is used throughout this document to refer to a defined characteristic of an individual — often referred to as subject attributes. Home address is one example of an attribute of a person. The term attribute value is used throughout to refer to a specifically assigned value for an attribute; for example, Jane Doe's home address is 1 Main St., Anytown, VA 1111. Attribute providers collect and maintain these elements - the attribute and its value[s] — together. In a federated environment, these attributes are asserted to the relying party (RP) to support the provision of a benefit or service, or when authorizing access to a protected resource.

Attributes and attribute values may also be associated with devices or non-person entities; however, these entities are not addressed in this document. For the purposes of this document, all attributes are deemed to be personally identifiable information (PII), and organizations should consider any security risks related to transmitting and retaining attributes, in addition to the various privacy risk considerations provided in this document.

Oftentimes, a set of asserted attributes and their values is enough on its own to support access to systems or applications. In the instance above, the information provided may be sufficient to allow Jane to benefit from a service her town provides for residents. Alternatively, in more sensitive contexts (e.g., national security systems, systems that enable access to personally identifiable information), RPs may want additional information about the specific attributes and attribute values they are receiving. Who provided Jane's home address? Did she self-assert it, or did the AP retrieve it from a database, such as the DMV or her employer? These data of the attributes, or metadata, enable the RP to interrogate the attribute value and information about the value itself during authorization policy evaluation. Information about the value may include where the attribute came from, whether it has been verified, and how often it is updated. This allows the RP to make a more informed decision about whether or not to trust an attribute when making access control decisions. Figure 1 illustrates the use of attribute metadata in an identity assertion.

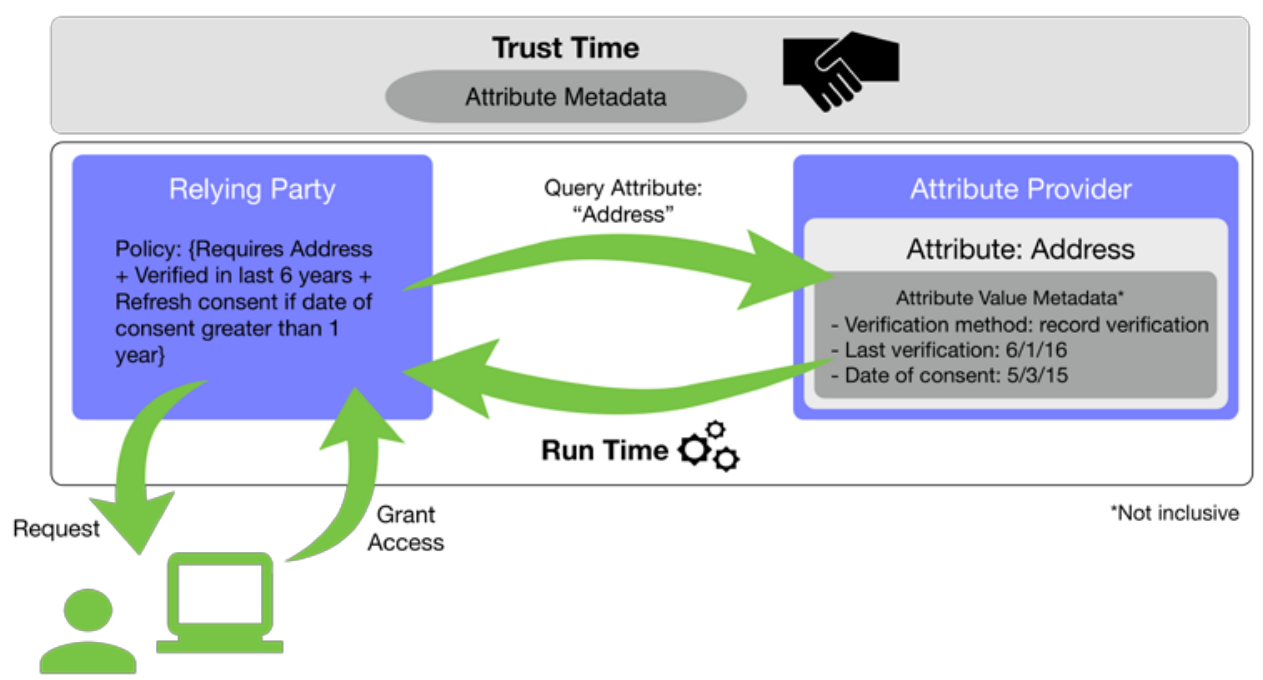

Figure 1 Trusted Access Using Attribute Metadata Assertion 
The ASM and AVM listed in each section of this schema are not mandatory. Collectively, these elements aim to support transactional needs in federations and trust frameworks across the private and public sectors. NIST envisions that communities and federations will leverage the included metadata elements to develop their own profiles (i.e., a tailored version of this schema). In addition, this schema supports extensibility for instances when the information contained herein is not sufficient for federated partners.

When implementing this schema, organizations must evaluate and understand both the authorization considerations and the privacy implications associated with a given use case or transaction type. With the additional granularity that attribute schema and attribute value metadata can provide, new information can be revealed which may provide a broader profile of an individual than was intended or anticipated. For instance, asserting that the verifier of Jane's address is her employer reveals more than just 1 Main St., Anytown, VA to the receiving entity. The receiving entity now knows who she works for in addition to where she resides, something that Jane may not be aware of and may not wish to reveal.

When deciding which metadata elements to select, the involved parties should conduct a privacy risk assessment to consider these possibilities, identify any potential adverse impacts to individuals' privacy that could arise from including certain metadata elements, and determine how to address identified privacy risks. To assist with this privacy risk assessment process, privacy considerations associated with the metadata elements are included throughout this document. This is certainly not an exhaustive list; there might be additional privacy considerations apart from those listed, and the listed considerations might change over time. Ultimately, the listed considerations may aid in deciding which elements of metadata to include, maximizing the benefit of a transaction while minimizing problems for the individuals associated with the metadata. If a metadata element does reveal information about an individual, but is still necessary to a transaction and thus must be included, the appropriate parties should consider how to provide visibility of the metadata to the individual or other measures to achieve predictability, manageability, or disassociability commensurate with the privacy risks introduced.

\subsection{Attribute Schema Metadata}

ASM provide information that is applicable to the attribute being asserted, regardless of the value of that attribute. ASM are intended to be static, discussed, and agreed upon by federated parties in advance of the actual assertion. For this reason, ASM are considered "trust-time" metadata and can be encapsulated in agreements such as attribute provider statements (APS), contracts, or trust frameworks. Table 3 provides the list of ASM that organizations can consider when establishing identity federation agreements. This is unlike AVM, which are dynamic and thus asserted and evaluated at run-time.

Table 3 - Attribute Schema Metadata

\begin{tabular}{|l|l|l|}
\hline \multicolumn{1}{|c|}{ Metadata } & \multicolumn{1}{c|}{ Description } & \multicolumn{1}{c|}{ Recommended Values } \\
\hline Description & An informative description of the attribute & Any \\
\hline
\end{tabular}




\begin{tabular}{|l|l|l|}
\hline Allowed Values & A defined set of allowed values for the attribute & Any \\
\hline Format & $\begin{array}{l}\text { A defined format in which the attribute will be } \\
\text { expressed }\end{array}$ & Any \\
\hline $\begin{array}{l}\text { Verification } \\
\text { Frequency }\end{array}$ & $\begin{array}{l}\text { The frequency at which the Attribute Provider } \\
\text { will re-verify the attribute }\end{array}$ & Any \\
\hline Data Processing & $\begin{array}{l}\text { Describes the condition for allowing the } \\
\text { processing of the attributes and attribute values }\end{array}$ & Any \\
\hline
\end{tabular}

\subsubsection{Description}

The description metadata element ensures that all entities participating in the federation of attributes have the same semantic understanding of the attribute. This enables both trust and interoperability by providing a common understanding of what the attribute and its value(s) represent. There are no set values for this metadata element as it is intended to be a free form, text-based definition.

\subsubsection{Allowed Values}

This metadata element provides a common, agreed-to set of values for an attribute. This ensures that when an AP transmits the attribute, the receiving organization is able to appropriately process the values. Variations between provider and RP in expressing values for an attributefor example, a value outside of an expected range-adversely impact interoperability and performance of authorization activities. For this reason, providing information for the resolution of this metadata element is highly recommended.

\subsubsection{Format}

This metadata element describes the format for expressing an attribute's value. For example, the attribute height may always be expressed in meters rather than centimeters. As with allowed values, up front agreement around the format of expressed attributes supports technical interoperability of assertions during run-time as well as appropriate policy evaluation of the attributes when determining access to resources.

\subsubsection{Verification Frequency}

In most situations, it is highly beneficial for the RP and the AP to agree to set rates for periodic verification of attribute values. This metadata element captures the frequency with which this reverification occurs, to ensure that both parties have established valid verification intervals. When 
determining if verification frequency is appropriate to include for a particular attribute, the parties should consider the fluidity of the attribute and its value; for example, date of birth may never need to be re-verified. They should also consider the risk associated with the transaction, or the environment in which the RP and AP are operating. Including this ASM element may negate the need for some of the currency AVM elements discussed later in this paper.

\subsubsection{Data Processing}

There may be legal requirements or trust framework policies that require a specified condition to be able to process data. Therefore, to avoid placing the RP in violation of any such requirements, it is beneficial for the AP and the RP to agree on whether a condition is required for the processing of the attributes before the attributes are sent to the RP and the type of condition required (e.g. subject consent, contract, legal obligation, public interest).

\subsection{Attribute Value Metadata}

While ASM are important, it is the granular attribute value metadata-for example, information about attribute values' authoritativeness, the processes used to create or establish them, and the frequency with which they are refreshed-that is designed to enable greater trust across systems. RPs can establish semantics and syntax of AVM at trust-time in order to make authorization decisions about access to resources or benefits at run-time. Regardless of the access control methodology leveraged by an organization, integrating AVM into decision support systems can enable more informed decisions and support richer policy development.

\subsubsection{Metadata Categories}

While AVM may be used for many purposes by RPs, certain metadata elements are more commonly tied to specific types of decisions. To facilitate RP decision-making and increase interoperability, this schema establishes five categories based on common uses of metadata: accuracy, currency, provenance, privacy, and classification. Each category of metadata elements is important for enabling the federation of attributes across a community or environment. Metadata associated with accuracy, currency, and provenance may facilitate crosssystem trust by establishing a consistent picture of the attribute value itself and the practices that generated that value, while privacy and classification can be leveraged to convey specific restrictions and protections that may need to be put in place based on certain data types, transactions, or use cases. Table 4 presents the five categories and their definitions. Table 5 provides a breakdown of the number of metadata elements by category.

The sections that follow list and provide details on the elements in each category.

Table 4 - Categories of Attribute Value Metadata

\begin{tabular}{|l|l|}
\hline Metadata Category & \multicolumn{1}{c}{ Description } \\
\hline Provenance & $\begin{array}{l}\text { Metadata relevant or pertaining to evaluating the source of the } \\
\text { attribute's value }\end{array}$ \\
\hline
\end{tabular}




\begin{tabular}{|l|l|}
\hline Metadata Category & \multicolumn{1}{c|}{ Description } \\
\hline Accuracy & $\begin{array}{l}\text { Metadata relevant or pertaining to determining if the } \\
\text { attribute's value is correct and belongs to a specific subject }\end{array}$ \\
\hline Currency & $\begin{array}{l}\text { Metadata relevant or pertaining to determining the “freshness" } \\
\text { of a given attribute's value }\end{array}$ \\
\hline Privacy & $\begin{array}{l}\text { Metadata relevant or pertaining to the management of privacy } \\
\text { policies relating to a given attribute's value }\end{array}$ \\
\hline Classification & $\begin{array}{l}\text { Metadata relevant or pertaining to the security classification of } \\
\text { a given attribute's value }\end{array}$ \\
\hline
\end{tabular}

Table 5 - Distribution of Attribute Value Metadata Elements

\begin{tabular}{|l|c|}
\multicolumn{1}{|c|}{ Metadata Category } & Number of Elements \\
\hline Provenance & 3 \\
\hline Accuracy & 2 \\
\hline Currency & 3 \\
\hline Privacy & 5 \\
\hline Classification & 2 \\
\hline All categories & 15 \\
\hline
\end{tabular}




\subsubsection{Provenance Metadata}

Table 6 - Provenance Metadata

\begin{tabular}{|c|c|c|}
\hline $\begin{array}{l}\text { Metadata } \\
\text { Element }\end{array}$ & Description & Recommended Values \\
\hline Origin & $\begin{array}{l}\text { The name of the entity that issues or creates } \\
\text { the initial attribute value }\end{array}$ & $\begin{array}{l}\text {-<Origin’s Name> } \\
\text {-“None” }\end{array}$ \\
\hline Provider & $\begin{array}{l}\text { The name of the entity that is providing the } \\
\text { attribute }\end{array}$ & $\begin{array}{l}\text {-<Provider’s Name> } \\
\text {-“None” }\end{array}$ \\
\hline Pedigree & $\begin{array}{l}\text { Description of the attribute value's } \\
\text { relationship to the authoritative source of } \\
\text { the value }\end{array}$ & $\begin{array}{l}\text {-“Authoritative” } \\
\text {-“Sourced” } \\
\text {-“Self-Asserted” } \\
\text {-“Derived” }\end{array}$ \\
\hline
\end{tabular}

\section{Origin}

The Origin element conveys the name of the entity that established the initial attribute value. This may or may not be an authoritative entity, or the provider; if, for example, the AP generates the attribute value through a derivation process, then the AP would be the origin. The key distinction between the origin and the provider is the act of initially generating, capturing, or provisioning the attribute's value, rather than just asserting the attribute's value to an RP.

\section{Provider}

This specifies the name of the entity that supplies the attribute value to the RP. This does not have to be the AP itself. This element enables RPs to understand and evaluate the source of the individual attribute values that may be included in a bundle of attributes. For example, if a full service credential provider generates an assertion with several identity attributes provided by multiple APs, the provider element enables the RP to understand, at a granular level, where each has come from and determine whether or not that value can be used for access to specific resources. In instances where a single attribute is asserted directly to the RP, this element may be omitted since the assertion itself will carry the provider information as well as a certificate or digital signature.

\section{Pedigree}

Pedigree refers to the attribute value's relationship to an authoritative source. Essentially, it allows the RP to understand better the process by which an attribute's value is generated and to determine whether or not it is from an acceptable authoritative source. Recommended values for this element include: 
1. Authoritative - The attribute's value was acquired directly from the source of authority. For example, an AP has received a driver's license number directly from the state DMV which issued the license.

2. Sourced - The attribute's value has been acquired from one or more non-authoritative sources. For example, an AP purchases a driver's license number from a third-party data aggregator.

3. Self-Asserted - The value was provided to the AP directly by the individual with whom the attribute value is associated. For example, an AP receives a driver's license number directly from the individual who references ownership of the license through a web form or questionnaire. Self-asserted attributes may also be verified or unverified.

4. Derived - The attribute value was produced through the analysis and manipulation of related attribute values and data. For example, a GPS ride sharing application could determine a value for a home address based on analysis of pick-up and drop-off locations. Derived should not be confused with the concept of Probabilistic Verification (a recommended value for the Verification Method), which focuses on verifying the attribute's value rather than generating it.

Taken in conjunction with the accuracy metadata, this information can enable the RP to better understand the origin of an attribute value, how it relates to its authoritative source, and how it has been verified - all of which help an RP establish a more complete picture of the value's usefulness and trustworthiness.

Privacy Considerations: Provenance metadata reveal information about the relationship between the data source and the subject which could allow for profiling of the subject beyond the purpose of authorization and which the subject may not know is occurring. For example, the origin value could reveal employment status and location, socio-economic information, or even health history; all of which may have unintended and potentially negative consequences for a subject's privacy.

Selection and use of these metadata elements should be carefully considered based on both authorization needs as well as a privacy risk assessment. For example, when leveraging attributes for access to moderate assurance level services that involve customers (i.e., non-enterprise users) it may be sufficient for the RP to request an attribute value's verification method without the origin element-the value of which may not outweigh the risk to privacy. The original source of the information may not be essential as long as the value has been verified using an acceptable method. To the extent selection of these elements is operationally necessary, RPs may manage the privacy risk through additional policies such as limiting use of the value outside of the authorization process or retaining the record of the verification without the actual value. 


\subsubsection{Accuracy Metadata Elements}

Table 7 - Accuracy Metadata

\begin{tabular}{|c|c|c|}
\hline $\begin{array}{l}\text { Metadata } \\
\text { Element }\end{array}$ & Description & Recommended Values \\
\hline Verifier & The entity that verified the attribute's value & $\begin{array}{l}\text {-“Origin” } \\
\text {-“Provider" } \\
\text {-“Not Verified” }\end{array}$ \\
\hline $\begin{array}{l}\text { Verification } \\
\text { Method }\end{array}$ & $\begin{array}{l}\text { The method by which the attribute value } \\
\text { was verified as true and belonging to the } \\
\text { specific individual }\end{array}$ & $\begin{array}{l}\text {-“Document Verification” } \\
\text {-“Record Verification” } \\
\text {-“Document Verification } \\
\text { with Record Verification" } \\
\text {-"Proof of Possession” } \\
\text {-"Probabilistic } \\
\text { Verification” } \\
\text {-"Not Verified” }\end{array}$ \\
\hline
\end{tabular}

\section{Verifier}

Verified attributes help RPs make informed decisions about whether to trust an attribute's value during policy evaluation. In addition, understanding who verified an attribute value may influence the RP's decision about whether or not to accept an attribute value as part of an access control decision. The verifier metadata element is intended to answer this "who" question. Namely, did the organization that established the attribute value perform the verification themselves or was the verification done at a later date by the AP? Acceptable values for this metadata field include:

1. Origin - The attribute's value was verified by the entity that issued or created it (e.g., a Social Security Number verified by the Social Security Administration).

2. Provider - The attribute's value was verified by the AP.

3. Not Verified - The value of the attribute was not verified.

\section{Verification Method}

This metadata element contains information on the process used to confirm that an attribute value is both true and belongs to the specified individual. This is sometimes necessary to support an authorization decision, but may not always be required. The acceptable values for verification method are intended to provide insight into the verification processes used by providers and enable greater confidence in a given attribute's value. This is particularly beneficial if there are multiple providers for instances of a single attribute. Recommended values for this element are: 
1. Document Verification - The attribute value was verified by inspecting a document that is acceptable to the RP (e.g., driver's license, medical record, utility bill). Transactional participants may want to determine the types of acceptable documents for attribute value verification in advance.

2. Record Verification - The attribute value was verified against an authoritative record or database. For the purposes of this schema, the term "authoritative" is used consistently with its definition in SP 800-63-3.

3. Document Verification with Record Verification - The attribute value was verified against both an acceptable document and an authoritative record or database.

4. Proof of Possession - Confirmation of an individual's ability to demonstrate possession of a device or account is used to verify the attribute's value. Certain attributes and their values, such as phone numbers and email addresses, can be verified by direct communication (SMS, voice, or email) with the entity to which the value is attributed. This method of verification may not be applicable to all attribute values. However, to a certain set of attributes, this is a legitimate approach to determining that the attribute's value is both valid and associated with the appropriate individual.

5. Probabilistic Verification - The AP has compared the attribute's value to multiple non-authoritative data sources to increase the probability that the attribute value is true and belongs to the appropriate individual. For example, rather than verifying a user address with the post office, an AP may compare the shipping addresses from multiple e-commerce transactions to increase confidence in the attributes' value. There may be many reasons an AP leverages "probabilistic verification" including the lack of available authoritative sources or limited automated capabilities to leverage authoritative sources. Methods of 'probabilistic verification' should be defined in the provider's APS and should be carefully considered for potentially negative privacy impacts. Probabilistic Verification should not be confused with the concept of a Derived pedigree, which focuses on generating the attribute's value rather than verifying it.

6. Not Verified - The attribute's value has not been verified.

\subsubsection{Currency Metadata}

Table 8 - Currency Metadata

\begin{tabular}{|l|l|l|}
\hline \multicolumn{1}{|c|}{$\begin{array}{c}\text { Metadata } \\
\text { Element }\end{array}$} & \multicolumn{1}{c|}{ Rescription } \\
\hline $\begin{array}{l}\text { Last } \\
\text { Verification }\end{array}$ & $\begin{array}{l}\text { The date and time when the attribute value } \\
\text { was last verified as being true and belonging } \\
\text { to the specified individual }\end{array}$ & No restrictions \\
\hline Last Refresh & $\begin{array}{l}\text { The date and time when the attribute was } \\
\text { last refreshed }\end{array}$ & No restrictions \\
\hline
\end{tabular}




\begin{tabular}{|l|l|l|}
\multicolumn{1}{|c|}{\begin{tabular}{c}
\multicolumn{1}{|c|}{ Metadata } \\
Element
\end{tabular}} & \multicolumn{1}{c|}{ Description } & Recommended Values \\
\hline $\begin{array}{l}\text { Expiration } \\
\text { Date }\end{array}$ & $\begin{array}{l}\text { The date an attribute's value is no longer } \\
\text { valid }\end{array}$ & No restrictions \\
\hline
\end{tabular}

\section{Last Verification}

RPs may not trust certain attribute values unless they have been verified within a certain time period. This is particularly true for certain values associated with attributes such as Role or Security Clearance, where the original established date of the value alone may not be sufficient for granting access to national security systems or data. Last Verification provides the most recent date and time at which the value was verified as true and belonging to the specified individual. This metadata provides only the last date that verification occurred, and does not include any information about method of verification.

\section{Last Refresh}

Last Refresh contains information on the date and time when an attribute's value was last refreshed. The age of the attribute can be derived from this attribute value. Last Refresh also allows RPs to determine the currency of the attribute value, and whether the attribute was updated recently enough to be used in a particular transaction.

\section{Expiration Date}

Attribute values sent from an AP to an RP may only be valid for its defined use for a set amount of time, depending on requirements, policy, or legal factors. The date after which an attribute's value is considered no longer valid for its defined use is the Expiration Date. Though Expiration Date and Last Refresh both allow an RP to determine if an attribute's value is current and sufficient, Expiration Date differs from Last Refresh in that there is a specified date or threshold after which the attribute's value becomes void for its defined use. RPs have the freedom to accept attributes after they have been considered expired for their original intended use, but this decision is made at their own discretion based upon the intended use of the attribute value, the type of interaction it is supporting, and the environment in which they operate. For example, an RP may choose to accept a recently expired driver's license number for access to a low assurance service. However, it is unlikely that an agency would accept a lapsed security clearance for access to classified data. 


\subsubsection{Privacy Metadata}

Table 9 - Privacy Metadata

Metadata

Description

Recommended Values

Element

\begin{tabular}{|l|l|l|}
\hline $\begin{array}{l}\text { Date of } \\
\text { Consent }\end{array}$ & $\begin{array}{l}\text { The date on which subject consent for release of } \\
\text { the attribute value was acquired }\end{array}$ & No restrictions \\
\hline Consent Type & Indicates the type of consent & No restrictions \\
\hline $\begin{array}{l}\text { Acceptable } \\
\text { Uses }\end{array}$ & $\begin{array}{l}\text { Allowed use conditions for entities that receive } \\
\text { attributes }\end{array}$ & No restrictions \\
\hline $\begin{array}{l}\text { Cache Time To } \\
\text { Live }\end{array}$ & $\begin{array}{l}\text { The length of time for which an attribute value } \\
\text { may be cached }\end{array}$ & No restrictions \\
\hline $\begin{array}{l}\text { Data Deletion } \\
\text { Date }\end{array}$ & $\begin{array}{l}\text { Indicates the date the attribute is to be deleted } \\
\text { from records }\end{array}$ & No restrictions \\
\hline
\end{tabular}

\section{Date of Consent}

As referenced in Subsection 3.1.5, the RP and AP may have agreed in advance on attribute metadata for conveying subject consent as a condition for processing the data when required by law or policy. In addition, some RPs may wish to understand when that consent was received; this could help an RP maintain predictability and manage any privacy risks arising from the duration of time between when the consent was initially granted and the current processing of the attributes.

\section{Consent Type}

As referenced in Subsection 3.1.5, and above in Date of Consent, the RP and AP may have agreed in advance on attribute metadata for conveying subject consent as a condition for processing the data when required by law or policy. In addition, some RPs may wish to understand the specific consent type where consent is a factor in maintaining predictability. For example, an RP may determine that a parental-delegated consent is a contextual factor that alters its analysis when assessing the privacy risk that may arise from the processing of the attributes. As a result, the RP can take appropriate steps to manage any identified privacy risks. Potential values for this element include, but are not limited to: opt-in, opt-out, parental-delegated, power of attorney-delegated. 


\section{Acceptable Uses}

This metadata element explains to receiving entities the use conditions for the attribute. For example, values might be limited to use for authorization, eligible for secondary uses beyond authorization, or not eligible for any further disclosure. Additionally, organizations or trust frameworks might also maintain their own categories of acceptable uses based on their policies. Potential values for this element include:

1. Authorization - The attribute value may only be used for processes related to authorization or compliance with law or legal process.

2. Secondary Use - The attribute value may be used for purposes beyond processes related to authorization or compliance with law or legal process. Secondary use may be unlimited, or subjects may only have agreed to specific types of uses such as service improvement, marketing, etc. Entities may choose as a best practice - or may be required by law or policy - to request separate, explicit consent from the user at initiation of the use.

3. No Further Disclosure - Although certain types of secondary uses by the RP may be permitted, further release to other third parties may not be permitted (unless required by law or legal process or unless with additional consent from the subject).

\section{Cache Time to Live}

This metadata element describes the length of time for which a specific attribute value may reside in cache memory for use again in future transactions. Due to the sensitivity of certain attributes' values, this metadata element enables the parties involved to cache properly and handle the values they are sending and retrieving as part of their transactions. In some cases, the time to live may be dictated by regulation or law, and this information needs to be relayed to RP systems so data are handled accordingly. The more sensitive an attribute value, the shorter time it will likely be enabled to live in temporary memory.

Note: Attribute value sensitivity cannot be treated as an absolute metric. Sensitivity is a contextual, risk-based determination. Therefore, even if an AP determines that the attribute value is not sensitive within the context of its system, receiving entities should make their own periodic risk assessments as to the attribute value's sensitivity based on the context of their systems, uses, and aggregation of additional data.

\section{Data Deletion Date}

This metadata element refers to long-term holding of attribute values. Minimizing data, and indicating the retention time for this data, is a generally accepted privacy principle. Some attribute values may produce little to no privacy risk for individuals. Other values may add new privacy risks or increase existing privacy risks. A deletion date ensures that sensitive information does not remain in systems indefinitely.

Note: Attribute value sensitivity cannot be treated as an absolute metric. Sensitivity is a contextual, risk-based determination. Therefore, even if an AP determines that within the 
context of its system, the attribute value is not sensitive, receiving entities should make their own periodic risk assessments as to the attribute value's sensitivity based on the context of their systems, uses, and aggregation of additional data.

\subsubsection{Classification Metadata}

Table 10 - Classification Metadata

\begin{tabular}{|c|c|c|}
\hline Metadata Element & Description & Recommended Values \\
\hline Classification & $\begin{array}{l}\text { The security classification level } \\
\text { of the attribute }\end{array}$ & $\begin{array}{l}\text {-“Unclassified” } \\
\text {-“Controlled Unclassified” } \\
\text {-“Confidential” } \\
\text {-“Secret” } \\
\text {-“Top Secret” } \\
\text {-“Company Confidential” }\end{array}$ \\
\hline Releasability & $\begin{array}{l}\text { The restrictions on who may } \\
\text { receive an attribute value }\end{array}$ & $\begin{array}{l}\text {-“NATO” } \\
\text {-“NOFORN” } \\
\text {-“FVEY” } \\
\text {-“Public Release” } \\
\text {-“Externally Releasable for Business } \\
\text { Purposes” } \\
\text {-“Do Not Release” } \\
\text {-“None” }\end{array}$ \\
\hline
\end{tabular}

\section{Classification}

Making certain attribute values available to RPs can carry national security implications. In these situations, identification of such attribute values at the time of exchange can be absolutely crucial to ensuring that the attribute values are appropriately handled and protected across the attribute's lifecycle. The recommended values for use in this schema are:

1. Unclassified - Unclassified attribute values are those that carry with them no national security implications. However, these attributes may still be sensitive and require special protections.

2. Controlled Unclassified - These attribute values are not sensitive enough to have a negative impact on national security, but are sensitive enough that they should be protected from improper access or exposure (e.g., For Official Use Only or "FOUO” information).

3. Confidential - Attribute values, which, if subject to unauthorized disclosure, could be expected to cause damage to national security.

4. Secret - Attribute values, which, if subject to unauthorized disclosure, could be expected to cause serious damage to national security. 
5. Top Secret - Attribute values, which, if subject to unauthorized disclosure, could be expected to cause exceptionally grave damage to national security.

6. Company Confidential - Attribute values which, if released, may cause damage to the organization, or the employees of the organization, that produced, generated, or maintains the values. For example, the professional title or specialization of a specific employee, if exposed, may inadvertently reveal information about a sensitive company project.

As with all classified information, the determination of the classification level for any attribute must be made by the appropriate authority, and the integrity of this classification must be maintained as the attribute and its values are transmitted or stored in by IT systems.

\section{Releasability}

Refers to restrictions that may be placed on the releasability of an attribute's value. The recommended values for this element include:

1. NATO - The attribute's value is releasable to North Atlantic Treaty Organization allies only and should not be distributed to other foreign nationals.

2. NOFORN - The attribute's value is not releasable to any foreign nationals.

3. FVEY - The attribute's value is releasable to Five Eye nations only.

4. Public Release - The attribute's value is explicitly approved for public release.

5. Externally Releasable for Business Purposes - The attribute's value has been explicitly approved for release to parties externally, but for approved business purposes only. For example, this may be leveraged by an entity to approve the release of attribute values as part of a federated environment supporting their supply chain.

6. Do Not Release - The attribute's value has not been approved for release beyond the originating organization.

7. None - There are no distribution or release caveats associated with the attribute's value. This, however, does not mean that the attribute value may be freely distributed. 


\section{$4 \quad$ Use Cases}

This section details three use cases as a means of demonstrating the ways in which ASM and AVM can be leveraged to enrich authorization decisions, facilitate cross boundary interoperability and trust, and enable adoption of federated attributes. Each use case carries with it a set of authorization and privacy considerations as well as suggested metadata necessary to fulfill evaluation of the requisite authorization policy, in addition to an example of what an AVM assertion may look like.

The use cases are:

1. Federated Access to Classified Documents in an Information Sharing Environment

2. Citizen Access to Federal Benefits

3. Law Enforcement Access to Intelligence Database

\subsection{Federated Access to Classified Documents in an Information Sharing Environment}

\section{Overview}

Monique, an Army employee with a current Secret clearance, attempts to access an information system that stores classified information, hosted by the Air Force on a shared Secure Internet Protocol Router Network (SIPRNet) site. Furthermore, the system, due to its sensitivity and the number of possible individuals that have legitimate need to access it, is protected using Attribute Based Access Control (ABAC) principles. ABAC evaluates access policy to enforce decisions based on attributes specific to the user and the resource (not addressed in the schema).

When Monique attempts access to the resource, an attribute query is routed to her agency, the Army, to obtain the attributes needed to grant or deny access. The Army then asserts the requested set of attributes, which are evaluated against the access control policy of the Air Force hosted site so a decision can be made.

While in an actual implementation there may be many different attributes required to access the protected resource, for the purposes of illustration, this use case will only focus on the clearance attribute. Furthermore, in this scenario it is assumed that the semantics and syntax associated with the attribute itself are established.

Table 11 - Clearance Attribute

\begin{tabular}{|l|l|}
\hline \multicolumn{2}{|c|}{ Attribute } \\
\hline Clearance & Secret \\
\hline
\end{tabular}

\section{Authorization Considerations}

In a traditional ABAC scenario, the assertion from the Army system would only provide the value that they maintain within their own records. As a result, the receiving agency's access 
control system is only able to make a decision based upon the asserted attribute value and nothing more (i.e., the employee's clearance is Secret therefore they are authorized for access). Information such as: the recency of the clearance issuance, when it was last verified by the asserting agency, and from where the value originated are not factored into the process.

With the inclusion of attribute metadata, the relying agency is able to make an informed, riskbased decision by adding the evaluation of the attribute metadata into their ABAC policies. For example, they could determine that anyone accessing this specific resource must have a Secret clearance that: originated from a DoD entity, has been verified in the last six months, and was verified by the providing entity against an authoritative database.

\section{Authorization Policy}

1. Origin MUST be an organization that is part of the Department of Defense

2. Verification of clearance MUST have been done in the last six months

3. Verification of clearance MUST have been done against an authoritative database

Through the establishment of AVM, these further considerations and requirements can be expressed in policy and compared to asserted information.

\section{Privacy Considerations}

In this scenario, the privacy risks are limited. Although the selected attributes are an absolute requirement for access based on national security needs, the requested value and metadata are minimal, and are being returned to a trusted party as part of the assertion in the context of the subject's employment duties.

\section{Suggested Attribute Value Metadata}

Based on the scenario's authorization and privacy considerations, the table below illustrates the AVM that is applied to support appropriate authorization decisions by the relying agency. It also provides notional values.

Table 12 - AVM to Support Access Control Decisions

\begin{tabular}{|c|c|}
\hline Element & Value \\
\hline Verifier & $\begin{array}{l}\text { Origin - The clearance was verified by the originating } \\
\text { entity - which in this case is the same as the provider }\end{array}$ \\
\hline Verification Method & $\begin{array}{l}\text { Record Check - The attribute value was verified against the } \\
\text { sponsoring agency's clearance database }\end{array}$ \\
\hline Last Verification & 6/10/16 (assume an access request date of 7/1/2016) \\
\hline
\end{tabular}




\begin{tabular}{|l|l|}
\hline \multicolumn{1}{|c|}{ Element } & \multicolumn{1}{c|}{ Value } \\
\hline Origin & United States Army \\
\hline Pedigree & $\begin{array}{l}\text { Authoritative - The attribute's value was generated and in } \\
\text { this case asserted as well by the authoritative source }\end{array}$ \\
\hline
\end{tabular}

\section{XACML Example Policy}

The following attribute and metadata names, and valid values, are fictional. These will ultimately depend on the technologies of the attribute sources that are being queried to evaluate policy. URIs and namespaces, in some cases, have been removed for brevity.

<xacml3:Policy Version="1.0" RuleCombiningAlgId="urn:oasis:names:tc:xacml:3.0:rule-combining-algorithm:de ny-overrides" PolicyId="http://www.axiomatics.com/automatic-unique-id/50f5b25e-dc7f-4672-a673-1a482e53f023 ">

<xacml3:Description>Use Case \#1</xacml3:Description>

<xacml3:PolicyDefaults><xacml3:XPathVersion>http://www.w3.org/TR/1999/REC-xpath-19991116</x acml3:XPathVersion $></$ xacml3:PolicyDefaults $>$

$<$ xacml3:Target/>

<xacml3:Rule RuleId="c01d7519-be21-4985-88d8-10941f44590a" Effect="Permit">

<xacml3:Description>isTSClearance</xacml3:Description>

$<$ xacml3:Target>

$<$ xacml3:AnyOf>

$<$ xacml3:AllOf>

<xacml3:Match MatchId="urn:oasis:names:tc:xacml:3.0:function:string-equal-ignore-case"> uteValue>

<xacml3:AttributeValue DataType="http://www.w3.org/2001/XMLSchema\#string">Secret</xacml3:Attrib

<xacml3:AttributeDesignator Category="urn:oasis:names:tc:xacml:1.0:subject-category:access-subject" Att ributeId="clearance.value" MustBePresent="false" DataType="http://www.w3.org/2001/XMLSchema\#string"/>

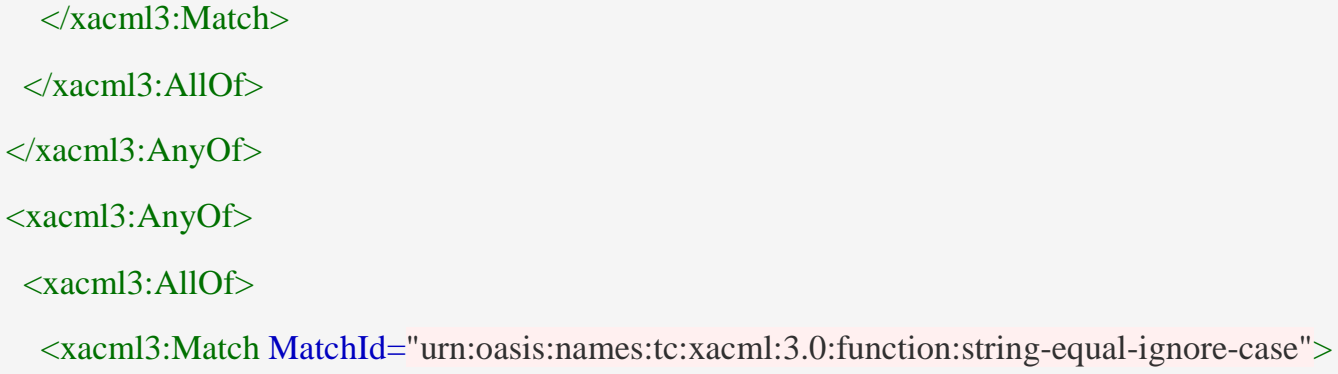



ributeValue>

<xacml3:AttributeValue DataType="http://www.w3.org/2001/XMLSchema\#string">ORIGIN</xacml3:Att

<xacml3:AttributeDesignator Category="urn:oasis:names:tc:xacml:1.0:subject-category:access-subject" Att ributeId="clearance.verifier" MustBePresent="false" DataType="http://www.w3.org/2001/XMLSchema\#string"/>

$</$ xacml3:Match>

$</$ xacml3:AllOf $>$

$<$ xacml3:AnyOf $>$

$<$ xacml3:AnyOf $>$

$<$ xacml3:AllOf $>$

<xacml3:Match MatchId="urn:oasis:names:tc:xacml:3.0:function:string-equal-ignore-case">

<xacml3:AttributeValue DataType="http://www.w3.org/2001/XMLSchema\#string">authoritative</xacml3 :AttributeValue>

<xacml3:AttributeDesignator Category="urn:oasis:names:tc:xacml:1.0:subject-category:access-subject" Att ributeId="clearance.pedigree" MustBePresent="false" DataType="http://www.w3.org/2001/XMLSchema\#string"/>

$</$ xacml3:Match>

$</$ xacml3:AllOf $>$

$<$ xacml3:AnyOf $>$

$<$ xacml3:AnyOf $>$

$<$ xacml3:AllOf>

<xacml3:Match MatchId="urn:oasis:names:tc:xacml:3.0:function:string-equal-ignore-case">

<xacml3:AttributeValue DataType="http://www.w3.org/2001/XMLSchema\#string">records check</xacml 3:AttributeValue>

<xacml3:AttributeDesignator Category="urn:oasis:names:tc:xacml:1.0:subject-category:access-subject" Att ributeId="clearance.verification_method" MustBePresent="false" DataType="http://www.w3.org/2001/XMLSchem a\#string"/>

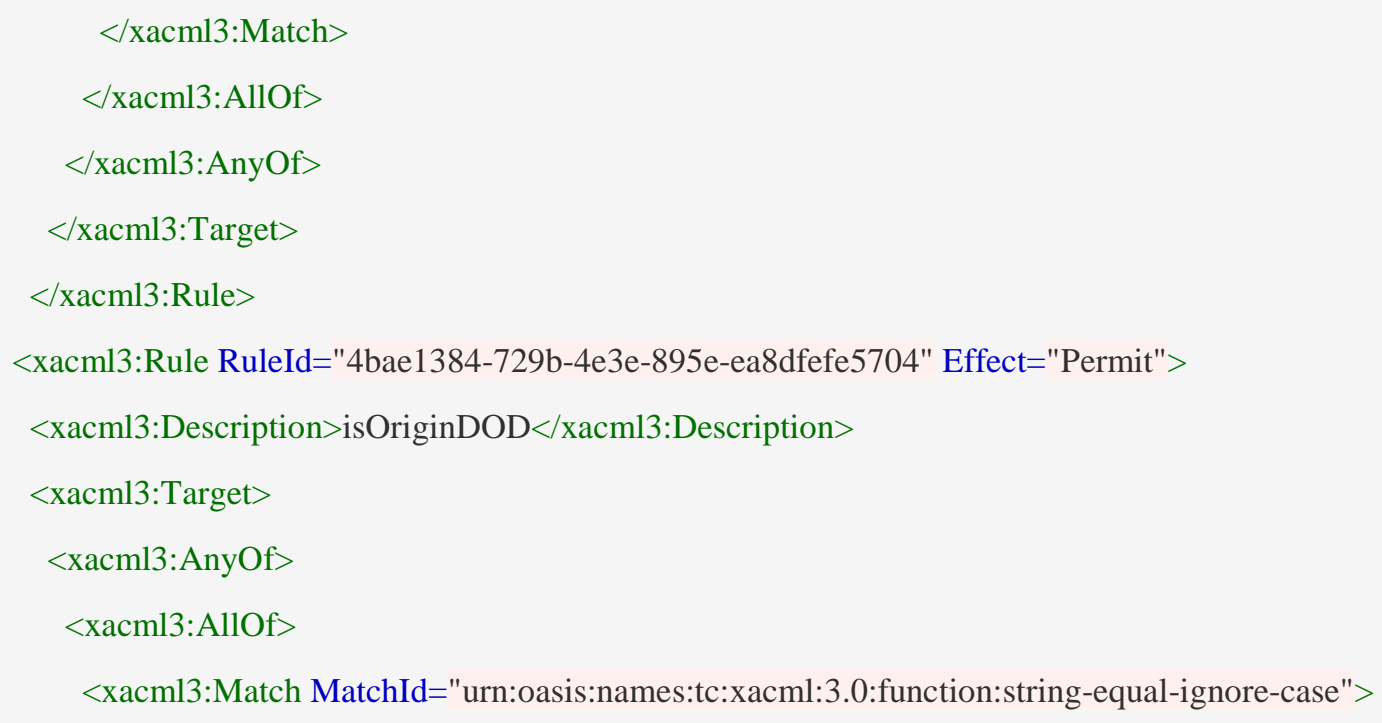




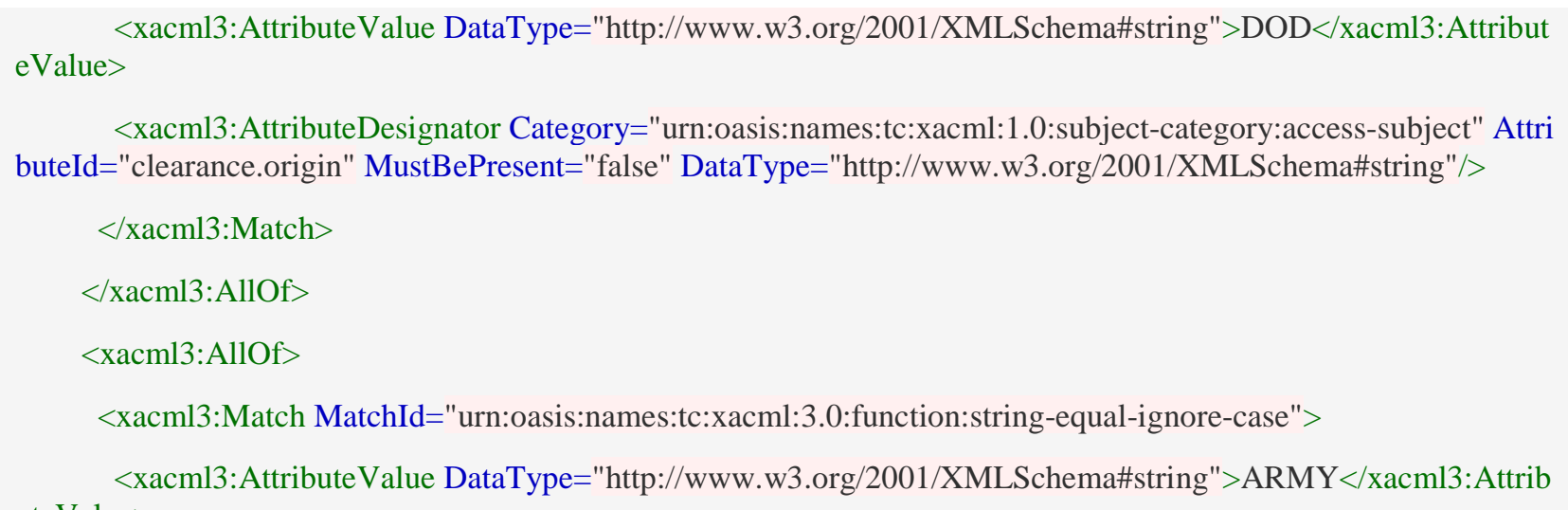

<xacml3:AttributeDesignator Category="urn:oasis:names:tc:xacml:1.0:subject-category:access-subject" Attri buteId="clearance.origin" MustBePresent="false" DataType="http://www.w3.org/2001/XMLSchema\#string"/>

$</$ xacml3:Match>

$</$ xacml3:AllOf $>$

$<$ xacml3:AllOf>

<xacml3:Match MatchId="urn:oasis:names:tc:xacml:3.0:function:string-equal-ignore-case">

<xacml3:AttributeValue DataType="http://www.w3.org/2001/XMLSchema\#string">MARINES</xacml3:A ttributeValue>

$<$ xacml3:AttributeDesignator Category="urn:oasis:names:tc:xacml:1.0:subject-category:access-subject" Attri buteId="clearance.origin" MustBePresent="false" DataType="http://www.w3.org/2001/XMLSchema\#string"/>

$</$ xacml3:Match>

$</$ xacml3:AllOf $>$

$<$ xacml3:AllOf $>$

<xacml3:Match MatchId="urn:oasis:names:tc:xacml:3.0:function:string-equal-ignore-case">

<xacml3:AttributeValue DataType="http://www.w3.org/2001/XMLSchema\#string">AIR FORCE</xacml3: AttributeValue $>$

$<$ xacml3:AttributeDesignator Category="urn:oasis:names:tc:xacml:1.0:subject-category:access-subject" Attri buteId="clearance.origin" MustBePresent="false" DataType="http://www.w3.org/2001/XMLSchema\#string"/>

$</$ xacml3:Match $>$

$</$ xacml3:AllOf $>$ 
$<$ xacml3:AllOf $>$

<xacml3:Match MatchId="urn:oasis:names:tc:xacml:3.0:function:string-equal-ignore-case">

<xacml3:AttributeValue DataType="http://www.w3.org/2001/XMLSchema\#string">USCG</xacml3:Attrib uteValue>

<xacml3:AttributeDesignator Category="urn:oasis:names:tc:xacml:1.0:subject-category:access-subject" Attri buteId="clearance.origin" MustBePresent="false" DataType="http://www.w3.org/2001/XMLSchema\#string"/>

$</$ xacml3:Match>

$</$ xacml3:AllOf $>$

$</$ xacml3:AnyOf $>$

$</$ xacml3:Target $>$

$</$ xacml3:Rule $>$

<xacml3:Rule RuleId="a17ecf55-77c0-4ddc-ab81-fcff342bcf7f" Effect="Permit">

$<$ xacml3:Description>verificationDateWithinYear</xacml3:Description>

$<$ xacml3:Target/>

<xacml3:Condition xmlns:xacml3="urn:oasis:names:tc:xacml:3.0:core:schema:wd-17">

<xacml3:Apply FunctionId="urn:oasis:names:tc:xacml:1.0:function:dateTime-less-than">

<xacml3:Apply FunctionId="urn:oasis:names:tc:xacml:1.0:function:dateTime-one-and-only">

<xacml3:AttributeDesignator Category="urn:oasis:names:tc:xacml:3.0:attribute-category:environment" Attri buteId="urn:oasis:names:tc:xacml:1.0:environment:current-dateTime" MustBePresent="false" DataType="http://w ww.w3.org/2001/XMLSchema\#dateTime"/>

$</$ xacml3:Apply $>$

<xacml3:Apply FunctionId="urn:oasis:names:tc:xacml:3.0:function:dateTime-add-yearMonthDuration">

<xacml3:Apply FunctionId="urn:oasis:names:tc:xacml:1.0:function:dateTime-one-and-only">

<xacml3:AttributeDesignator Category="urn:oasis:names:tc:xacml:1.0:subject-category:access-subject" Att ributeId="clearance.last_verification" MustBePresent="false" DataType="http://www.w3.org/2001/XMLSchema\#d ateTime"/>

$<$ xacml3:Apply>

<xacml3:AttributeValue DataType="http://www.w3.org/2001/XMLSchema\#yearMonthDuration">P6M</xa cml3:AttributeValue>

$</$ xacml3:Apply $>$

$<$ xacml3:Apply>

$</$ xacml3:Condition>

$</$ xacml3:Rule $>$

$</$ xacml3:Policy $>$ 


\subsection{Citizen Access to Federal Benefits}

\section{Overview}

Jane is a veteran, and she is in the process of establishing an online account to manage her Veterans Affairs (VA) educational benefits. The VA system leverages a federated identity model that is integrated with multiple trusted Identity Providers (IDPs), which offer high assurance credentials and identity attributes. Furthermore, the VA system leverages the asserted attributes to both populate the online registration form and to make an initial eligibility determination when establishing an account.

When Jane initiates the registration process she is notified by her IDP which attributes are being asserted to the VA, for what they are going to be used, and what type of metadata is being provided. Failure to enroll via the online process (if, for example the attribute value metadata is not within policy) triggers a backup offline verification process conducted by the VA.

Table 13 - Veteran Status Attribute

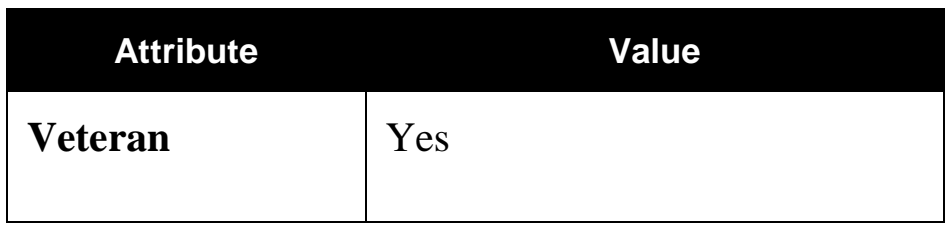

\section{Authorization Considerations}

For this transaction, the VA has identified the attribute Veteran Status as critical to making an initial authorization decision. Though the VA is likely to have an existing record for Jane, it may not be easily accessible to the application. To ease the process of online enrollment for the service, the VA has determined an external assertion of veteran status is sufficient to open an account if the following policy is met:

\section{Authorization Policy}

1. Veteran status must have been verified by the provider or the originating authority

2. Veteran status must have been verified through document verification and against an authoritative database

\section{Privacy Considerations}

In this use case, some metadata elements with privacy implications, such as provider, are necessary for the transaction. Adding additional controls to maintain predictability, manageability, and disassociability commensurate with identified privacy risks can better manage these privacy concerns. For example. since provider must be included, obtaining explicit consent from Jane before releasing her veteran status (as required by the authorization policy), enables Jane to be aware of the transfer of this attribute value, and to grant her permission for the transfer or decline the transaction based on her preference. Other metadata elements with privacy implications, such as origin, are not needed in this transaction, technically or policy-wise. Thus, 
they should be excluded since they are not necessary and their inclusion would potentially reveal a broad profile of Jane (e.g., related to her associations with certain organizations).

\section{Suggested Attribute Value Metadata}

Based on the scenario's authorization and privacy considerations, the table below illustrates the AVM that is applied to support appropriate decisions by the VA system. It also provides notional values.

Table 14 - AVM to Support Enrollment Decisions

\begin{tabular}{|l|l|}
\hline \multicolumn{1}{|c|}{ Element } & $\begin{array}{l}\text { Value } \\
\text { Verifier } \\
\text { acting as the AP in this instance) }\end{array}$ \\
\hline $\begin{array}{l}\text { Verification } \\
\text { Method }\end{array}$ & $\begin{array}{l}\text { Document verification with Record Check - The attribute } \\
\text { value was verified against a DD-214 provided by Jane and } \\
\text { was checked against a National Archives and Records } \\
\text { Administration database }\end{array}$ \\
\hline
\end{tabular}

\section{XACML Example Policy}

The following attribute and metadata names, and valid values, are fictional. These will ultimately depend on the technologies of the attribute sources that is being queried to evaluate policy. URI's and namespaces, in some cases, have been removed for brevity.

<xacml3:Policy Version="1" RuleCombiningAlgId="urn:oasis:names:tc:xacml:3.0:rule-combining-algorithm:denyoverrides" PolicyId="9458137c-535b-4f2e-9907-2e8c7d5881ad">

<xacml3:Description>Use Case \#2</xacml3:Description>

<xacml3:PolicyDefaults>

<xacml3:XPathVersion>http:/www.w3.org/TR/1999/REC-xpath-19991116</xacml3:XPathVersion>

$</$ xacml3:PolicyDefaults $>$

$<$ xacml3:Target/>

<xacml3:Rule RuleId="35e6f270-5504-4596-9786-431d7de04402" Effect="Permit">

<xacml3:Description>isVeteran</xacml3:Description>

$<$ xacml3:Target>

$<$ xacml3:AnyOf $>$ 
$<$ xacml3:AllOf $>$

<xacml3:Match MatchId="urn:oasis:names:tc:xacml:1.0:function:boolean-equal">

<xacml3:AttributeValue DataType="http://www.w3.org/2001/XMLSchema\#boolean">true</xacml3:Attribu teValue $>$

<xacml3:AttributeDesignator Category="UC2" AttributeId="veteran.value" MustBePresent="false" DataTyp e="http://www.w3.org/2001/XMLSchema\#boolean"/>

$</$ xacml3:Match>

$</$ xacml3:AllOf $>$

$</$ xacml3:AnyOf $>$

$<$ xacml3:AnyOf >

$<$ xacml3:AllOf $>$

<xacml3:Match MatchId="urn:oasis:names:tc:xacml:3.0:function:string-equal-ignore-case" >

<xacml3:AttributeValue DataType="http://www.w3.org/2001/XMLSchema\#string">PROVIDER</xacml3: AttributeValue $>$

$<$ xacml3:AttributeDesignator Category="UC2" AttributeId="veteran.verifier" MustBePresent="false" DataT ype="http://www.w3.org/2001/XMLSchema\#string"/>

$</$ xacml3:Match $>$

$</$ xacml3:AllOf $>$

$</$ xacml3:AnyOf $>$

$<$ xacml3:AnyOf $>$

$<$ xacml3:AllOf $>$

<xacml3:Match MatchId="urn:oasis:names:tc:xacml:3.0:function:string-equal-ignore-case">

<xacml3:AttributeValue DataType="http://www.w3.org/2001/XMLSchema\#string">\&gt;Document Verific ation with Record Verification</xacml3:AttributeValue $>$

$<$ xacml3:AttributeDesignator Category="UC2" AttributeId="veteran.verification_method" MustBePresent=" false" DataType="http://www.w3.org/2001/XMLSchema\#string"/>

$</$ xacml3:Match $>$

$</$ xacml3:AllOf $>$

$<$ /xacml3:AnyOf $>$

</xacml3:Target $>$

</xacml3:Rule>

$</$ xacml3:Policy $>$ 


\subsection{Law Enforcement Access to a Government Database}

\section{Overview}

Claude is with the Los Angeles Police Department (LAPD) and is attempting to access an FBI criminal justice database to gather additional information for a high-profile case. This database uses a federated identity model with multiple IDPs across affiliated law enforcement agencies. Due to the sensitive information retained within the database, access is protected based on ABAC. The attributes are asserted by the appropriate law enforcement agency (in this case the LAPD) to the FBI, who is then able to evaluate the attributes and make an access decision.

Table 15 - Law Enforcement Officer Attributes

\begin{tabular}{|l|l|}
\hline \multicolumn{2}{|c|}{ Attribute } \\
\hline Sworn Law Enforcement Officer & Yes \\
\hline CJIS Privacy Training & Yes \\
\hline
\end{tabular}

\section{Authorization Considerations}

We assume in this example that the access request was sent on 7/1/16. The FBI allows access to this database based on two major requirements. The first requirement is that Claude must be a Sworn Law Enforcement Officer (LEO), verified at least quarterly to prevent granting access to retired users. The second requirement is that Claude must have completed Criminal Justice Information System (CJIS) Privacy Training. This training must have been completed - and verified - within the last 12 months.

\section{Authorization Policy}

1. Origin MUST be FBI or an affiliated law enforcement agency

2. User MUST be a Sworn LEO with status validated within the last quarter (3 months)

3. CJIS Privacy Training MUST have been completed within the last 12 months

\section{Privacy Considerations}

In this use case, certain metadata elements are necessary to demonstrate compliance with access requirements for this database. However, excessive metadata collection that extends beyond these requirements could unnecessarily reveal information about law enforcement officials accessing the system. For example, provider metadata is not necessary for this transaction, and could reveal unintended information about Claude by divulging his relationship with the provider organization. Other metadata elements (e.g., origin) are necessary, but might still have privacy implications for Claude by revealing information about him. In these instances, it is important to-when possible - ensure that Claude is aware of which information is being transferred. 


\section{Suggested Attribute Value Metadata}

Based on the scenario's authorization and privacy considerations, the table below illustrates the AVM that is applied to support appropriate authorization decisions by the FBI. It also provides notional values.

Table 16 - AVM to Support Federated Access Decisions

\begin{tabular}{|c|c|}
\hline Element & Value \\
\hline Verifier & $\begin{array}{l}\text { Origin - The status and verification dates for both } \\
\text { Sworn LEO and CJIS Privacy Training would be } \\
\text { verified by the originating entity (LAPD) }\end{array}$ \\
\hline $\begin{array}{l}\text { Last Verification (Sworn } \\
\text { LEO) }\end{array}$ & 6/15/16 \\
\hline $\begin{array}{l}\text { Last Verification (CJIS } \\
\text { Privacy Training) }\end{array}$ & 6/1/15 \\
\hline Origin & Los Angeles Police Department \\
\hline Pedigree & $\begin{array}{l}\text { Authoritative - The attribute's value was generated } \\
\text { and in this case asserted as well by the authoritative } \\
\text { source }\end{array}$ \\
\hline
\end{tabular}

Based on information about the user sent to the FBI by the LAPD IDP, the user is a Sworn LEO and has been verified as such within the last month (6/15/16). The user has also completed CJIS Privacy Training. However, the last verified date for the CJIS Privacy Training value was 13 months ago (6/1/15). In accordance with policy and based on interrogation of AVM, Claude is denied access based on the amount of time since the value for CJIS Privacy Training was verified. Here, the FBI has maintained its policy that simply taking the CJIS Privacy Training is not enough; it must have also been completed and verified within the last year as well. Similar to the "Federated Access to Classified Document in an Information Sharing Environment" example, the inclusion of AVM allows for more informed and fine-grained access control decisions than in a traditional ABAC instance.

\section{XACML Example Policy}

The following Attribute and metadata names, and valid values, are fictional. These will ultimately depend on the technologies of the attribute sources that are being queried to evaluate policy. URI's and namespaces, in some cases, have been removed for brevity. 
<xacml3:Policy Version="1" RuleCombiningAlgId="urn:oasis:names:tc:xacml:3.0:rule-combining-algorithm:denyoverrides" PolicyId="72537098-66a9-4283-8790-9c567eb2be1d">

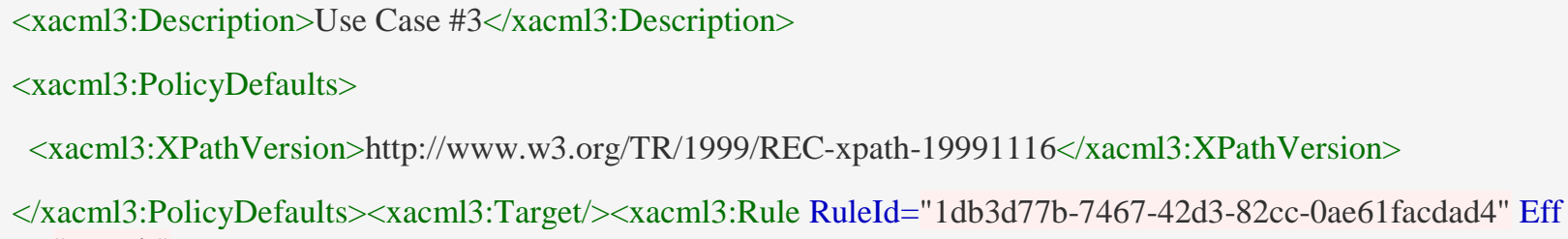




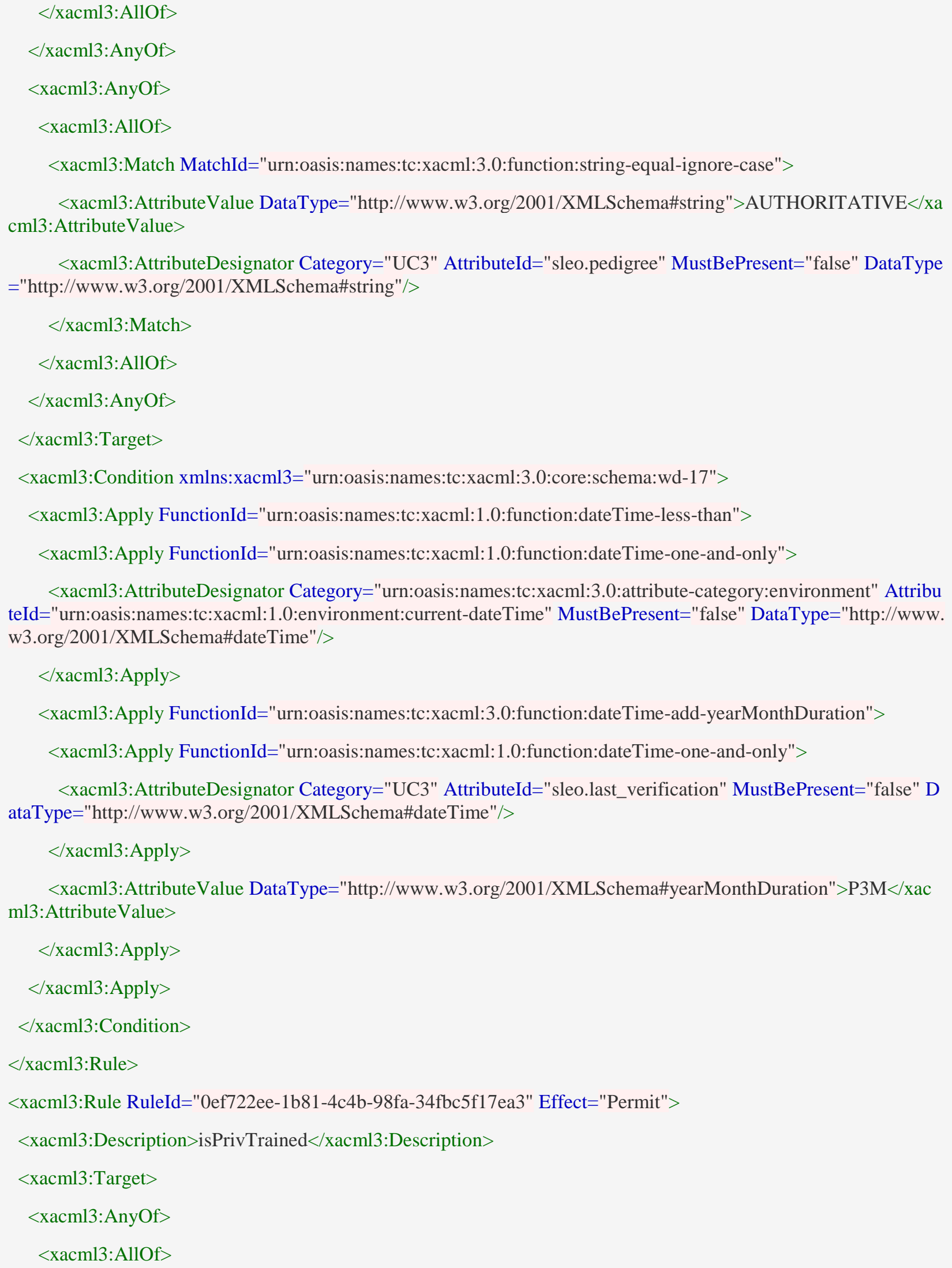


<xacml3:Match MatchId="urn:oasis:names:tc:xacml:1.0:function:boolean-equal">

<xacml3:AttributeValue DataType="http://www.w3.org/2001/XMLSchema\#boolean">true</xacml3:Attribut eValue $>$

<xacml3:AttributeDesignator Category="UC3" AttributeId="cjis_privacy_training.value" MustBePresent="fa lse" DataType="http://www.w3.org/2001/XMLSchema\#boolean"/>

$</$ xacml3:Match>

$</$ xacml3:AllOf $>$

$</$ xacml3:AnyOf $>$

$<$ xacml3:AnyOf $>$

$<$ xacml3:AllOf $>$

<xacml3:Match MatchId="urn:oasis:names:tc:xacml:3.0:function:string-equal-ignore-case">

<xacml3:AttributeValue DataType="http://www.w3.org/2001/XMLSchema\#string">ORIGIN</xacml3:Attrib uteValue>

<xacml3:AttributeDesignator Category="UC3" AttributeId="cjis_privacy_training.verifier" MustBePresent=" false" DataType="http://www.w3.org/2001/XMLSchema\#string"/>

$</$ xacml3:Match $>$

$</$ xacml3:AllOf $>$

$</$ xacml3:AnyOf $>$

$<$ xacml3:AnyOf $>$

$<$ xacml3:AllOf $>$

<xacml3:Match MatchId="urn:oasis:names:tc:xacml:3.0:function:string-equal-ignore-case">

<xacml3:AttributeValue DataType="http://www.w3.org/2001/XMLSchema\#string">LAPD</xacml3:Attribut eValue>

<xacml3:AttributeDesignator Category="UC3" AttributeId="cjis_privacy_training.origin" MustBePresent="fa lse" DataType="http://www.w3.org/2001/XMLSchema\#string"/>

$</$ xacml3:Match>

$</$ xacml3:AllOf $>$

$</$ xacml3:AnyOf $>$

$<$ xacml3:AnyOf $>$

$<$ xacml3:AllOf $>$

<xacml3:Match MatchId="urn:oasis:names:tc:xacml:3.0:function:string-equal-ignore-case" >

$<$ xacml3:AttributeValue DataType="http://www.w3.org/2001/XMLSchema\#string">AUTHORITATIVE $<$ xa cml3:AttributeValue>

<xacml3:AttributeDesignator Category="UC3" AttributeId="cjis_privacy_training.pedigree" MustBePresent= "false" DataType="http://www.w3.org/2001/XMLSchema\#string"/>

$</$ xacml3:Match>

$<$ xacml3:AllOf $>$ 
$</$ xacml3:AnyOf $>$

$</$ xacml3:Target $>$

<xacml3:Condition xmlns:xacml3="urn:oasis:names:tc:xacml:3.0:core:schema:wd-17">

<xacml3:Apply FunctionId="urn:oasis:names:tc:xacml:1.0:function:dateTime-less-than">

<xacml3:Apply FunctionId="urn:oasis:names:tc:xacml:1.0:function:dateTime-one-and-only">

<xacml3:AttributeDesignator Category="urn:oasis:names:tc:xacml:3.0:attribute-category:environment" Attribu teId="urn:oasis:names:tc:xacml:1.0:environment:current-dateTime" MustBePresent="false" DataType="http://www. w3.org/2001/XMLSchema\#dateTime"/>

$</$ xacml3:Apply $>$

<xacml3:Apply FunctionId="urn:oasis:names:tc:xacml:3.0:function:dateTime-add-yearMonthDuration">

<xacml3:Apply FunctionId="urn:oasis:names:tc:xacml:1.0:function:dateTime-one-and-only">

<xacml3:AttributeDesignator Category="UC3" AttributeId="cjis_privacy_training.last_verification" MustBeP resent="false" DataType="http://www.w3.org/2001/XMLSchema\#dateTime"/>

$<$ xacml3:Apply>

<xacml3:AttributeValue DataType="http://www.w3.org/2001/XMLSchema\#yearMonthDuration" $>$ P1Y $</$ xacm 13:AttributeValue>

$<$ xacml3:Apply $>$

</xacml3:Apply>

</xacml3:Condition>

</xacml3:Rule>

$</$ xacml3:Policy $>$ 


\section{References}

[SP 800-162] NIST Special Publication 800-162, Guide to Attribute Based Access Control Definition and Considerations, January 2014. Available at:

http://nvlpubs.nist.gov/nistpubs/specialpublications/NIST.sp.800-162.pdf.

[SP 800-63-3] DRAFT NIST Special Publication 800-63-3, Digital Identity Guidelines, January 2017. Available at: https://pages.nist.gov/800-63-3/. 